\title{
Memory Models for Improving Tabu Search with Real Continuous Variables
}

\author{
Dr Andrew M. Connor \\ Software Engineering Research Lab, Auckland University of Technology \\ andrew.connor@aut.ac.nz
}

\begin{abstract}
This paper proposes that current memory models in use for tabu search algorithms are at best evolving, as opposed to adaptive, and that improvements can be made by considering the nature of human memory. By introducing new memory structures, the search method can learn about the solution space in which it is operating. The memory model is based on the transfer of events from episodic memory into generalised rules stored in semantic memory. By adopting this model, the algorithm can intelligently explore the solution space in response to what has been learned to date and continuously update the stored knowledge.
\end{abstract}

\section{Introduction}

Global optimisation algorithms such as genetic algorithms and simulated annealing have attracted attention in recent years as a means of solving complex problems. Although these have been shown to be capable of solving problems that are otherwise intractable, there is a growing interest in other "heuristic" algorithms such as tabu search [1,2].

Previous work $[3,4,5]$ has led to the development of a mature algorithm for solving problems with real, continuous variables. Whilst the implementation is somewhat different from the original tabu search concept, results on a wide range of optimisation problems have shown the algorithm to be both effective and efficient. It is the aim of this paper to tentatively propose an approach that, once implemented, may further improve the efficiency of the method for problems with large numbers of variables. One of the strengths of the tabu search approach is that utilises search history information to guide the future search vector. This paper presents a proposal to implement an added layer of intelligence based on learning from search history to further enhance the method.

\section{Tabu search}

Tabu search is a metaheuristic which is used to guide optimisation algorithms in the search for a globally optimal solution. The tabu search algorithm uses flexible memory cycles of differing time spans to force the search out of local optima and to provide strategic control of how the search progresses through the solution space. Tabu search is classed as a metaheuristic as it is an added layer of control added to any local search method. Local search employs the idea that a given solution $\mathrm{S}$ may be improved into a new solution $S^{\prime}$ by making a small change. The solution $S^{\prime}$ is chosen by evaluating the neighbours of $S$ according to an objective function and selecting the neighbour with the best objective function value. The local search algorithm starts with some initial solution and moves from neighbour to neighbour as long as possible while decreasing the objective function value. The tabu search metaheuristic allows a local search method to escape local optima into which it descends.

Tabu search is based on the premise that for a search method to be classed as "intelligent" it must incorporate adaptive memory and responsive exploration. The use of adaptive memory enables the searching of solution space efficiently and effectively. Since local choices are guided by information collected during the search, there is a distinct contrast between tabu search and semirandom, memoryless approaches such as genetic algorithms and simulated annealing. Adaptive memory also contrasts with rigid memory designs typical of branch and bound strategies. The emphasis on responsive exploration in tabu search derives from the belief that a bad strategic choice can yield more information about a solution space than a good random choice. In a memory based system, a bad choice based on a particular strategy can provide potential clues about how the strategy may be improved. Responsive exploration integrates the basic principles of intelligent search, i.e., exploiting good solution features while exploring new promising regions. Tabu search is concerned with finding new 
and more effective ways of taking advantage of the mechanisms associated with both adaptive memory and responsive exploration.

\subsection{Adaptive Memory}

The term "adaptive memory" underlies the concept of learning in tabu search, where different kinds of memory are integrated advantageously. This is especially true of integrating recency based and frequency based memory. Recency based memory keeps track of solution parameters that have changed in the search history whereas frequency based keeps track off of the number of times each particular parameter is changed or the number of times each parameter is represented in a recency based memory. Both recency and frequency based memory can be implemented to both "elite" solutions or to all solutions.

\subsection{Current Memory Implementation}

The tabu search method on which current work is based has been implemented with a number of different memories as detailed in Table 1. Each memory has a different type and purpose.

Table 1. Implemented memories

\begin{tabular}{lll}
\hline \hline Memory & Type & Purpose \\
\hline Short Term & Recency & $\begin{array}{l}\text { Avoid cycling } \\
\text { around local optima }\end{array}$ \\
\hline $\begin{array}{l}\text { Intermediate } \\
\text { Term }\end{array}$ & Recency & $\begin{array}{l}\text { Reinforce “good” } \\
\text { characteristics }\end{array}$ \\
\hline Long Term & Recency & Expand search focus \\
\hline Parameter & Frequency & $\begin{array}{l}\text { Improve search } \\
\text { efficiency }\end{array}$ \\
\hline \hline
\end{tabular}

The short term memory is a recency based memory that modifies the search vector by recognising "tabu conditions”. Figure 1 illustrates a simple search vector.

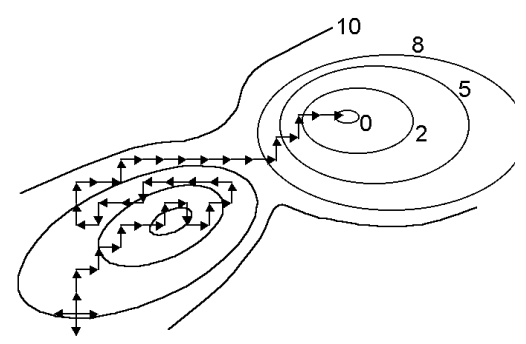

Figure 1. Search Vector

A tabu condition prevents a particular solution, or set of solutions, from being chosen as the outcome of the next move. The tabu status remains valid for a certain number of iterations and so the set of tabu solutions is essentially the last " $n$ " visited solutions. This has the effect of limiting the method from cycling around local optima as shown in Figure 1, where the search vector tracks between two local optima as it cannot return to previously visited solutions.

The intermediate memory is also recency based, but rather than record a set of recently visited solutions it is a set of the last visited "elite" solutions. An elite solution is one that is better than the previously best found solution. Elite solutions are found infrequently, so this memory has a longer time scale. By periodically examining the elite solutions set it is possible to determine whether good solutions have features in common. These features can then be reinforced to intensify the search in order to find new elite solutions.

The long term memory consists of a set of solutions sampled at fixed periods. These solutions therefore represent different areas of the solution space. This is a crude representation of the full search vector which can be used to direct a search to new areas once elite solutions in the current locale are not being discovered.

Finally, the parameter memory is used to improve the efficiency of the search. This memory is frequency based and is in indication of how often each solution parameter is changed when moves are made in the solution space. When a parameter is changed often in quick succession, it implies that the solution space has consistent characteristics and is likely to be continuous and non-multi modal. When this condition exists, rather than explore all solutions in the vicinity of the current locale, the frequently changed parameter is changed repeatedly as long as local improvement continues to be found.

Whilst the combination of the various memories has led to a very robust optimisation tool, it is questionable whether the memories should really be considered "adaptive". At best, they are responsive and evolving. For recency based memory, they can only contain specific knowledge with regards the solution space for as long as specific solutions are retained in the memory set. Similarly, for frequency based memory, as the search vector moves through the solution space it is possible that knowledge based on frequency of parameter changes may become inappropriate. Here, at least, as new frequency data is amassed it can give rise to "valid" knowledge again.

The conjecture of this paper is that an examination of the memory processes in the human mind may give rise to better memory models for use in heuristic search. In particular the intent of future work is to discover ways that search information can be stored 
and utilised to overcome the inefficiency of the underlying local search methods. Local search becomes exceptionally inefficient when there are a large number of search variables as it is necessary to evaluate all neighbouring solutions to the current solution in order to be able to select the best move. By reconsidering how search information is stored and utilised it may be feasible for only a subset of the neighbouring solutions to be evaluated, therefore vastly improving the efficiency of the search

\section{Proposed Memory Model}

The basic recency based memory structures used in the tabu search implementation are similar to those described in the Atkinson-Shiffrin model [6] which categorise memory according to the amount of time it is stored in the brain. The first type of memory is sensory memory which contains information received immediately from a person's senses into the human brain. Sensory information is stored for just an instant in sensory registers in the brain and is relatively unprocessed. What processing that occurs determines which information can become a focus for further consideration or processing.

Short term memory in individuals is a working memory that can retain around seven "chunks" of information for retrieval for up to thirty seconds after which information is lost if it is not repeated or rehearsed. Information that remains in the brain for more than a few minutes is encoded into the brain by being transferred to long term memory. Long term memory allows information to be retrieved even after it has been stored in the brain and out of human consciousness for a long period of time [7].

It has been said that long term memory is what "defines reality for each of us" [7]. Its capacity is believed to be unlimited. Information in long term memory could have been learned, or encoded, five minutes ago or five decades ago. Some scientists believe that such information is stored permanently whereas others argue that with time memory pathways will physically deteriorate over time. Information in long term memory can be classified according to what is being remembered. Tulving's multiple memory model $[8,9]$ categorises memory into episodic memory, semantic memory and procedural memory. Episodic memory, or autobiographical memory, is an explicit memory of events that have occurred. In the human mind, this includes time, place and associated emotions which affect the quality of the memory. Episodic memory contrasts and interacts with semantic memory, the memory of facts and concepts. Semantic memory refers to the memory of meanings, understandings, and other knowledge; in contrast to episodic memory. It is typically viewed as general world-knowledge such as vocabulary, grammar or "facts".

An important difference between the two is that semantic memories are relatively bereft of context, they appear to the individual simply as known facts, whereas episodic memories involve significant components of context in which the memory was formed. Episodic and semantic memory are both classified as types of declarative memory because people can consciously recall facts, events, and experiences and then verbally declare or describe their recollections. In contrast, nondeclarative or procedural, memory is expressed through performance and typically does not require a conscious effort to recall. Procedural memory is the long-term memory of skills and procedures, or "how to" knowledge. The concept of procedural memory is no considered in this paper.

It is believed that long term memory begins as episodic memory, stored in the limbic structures of the brain. This episodic memory carries with it an involvement of the self in the relevant episode or episodes. Then, through a sequence of re-workings, memory may be transferred to semantic memory, situated in the associative areas of the neo-cortex. Episodic memories therefore can be amalgamated into semantic memory, making them part of our accumulated experience and broaden general understanding of the world.

In future work, it is proposed to introduce the concepts of both episodic and semantic memory into the long term memory used in the tabu search implementation. The aim is to add a subset to the longer term memory called selection memory, which will hold knowledge that enables the selection of a subset of the neighbours of the current search point to be selected rather than evaluating all of the neighbours of the current locale, S. Figure 2 illustrates the structure of the memories associated with an individual search thread. 


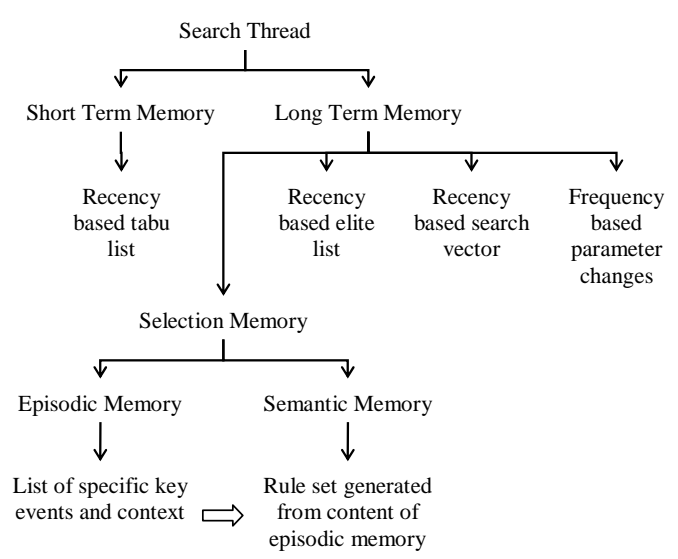

Figure 2. Proposed Memory Model

In the proposed implementation, the semantic memory will store key events that occur throughout the search of the solution space. This is distinct from the concept of storing the location of elite solutions in a recency list. Key events can also include the discovery of sensitivity of performance to parameter changes, the location of discontinuities in the solution space and so forth. As the search progresses, events in episodic memory will be analysed for common attributes from which general rules can be created. These rules will be applied to the selection of a subset of the current neighbourhood to evaluate for each move.

Because the implementation of tabu search used is designed to work with continuous real variables there is a significant challenge in understanding how key events in the search history may be represented and stored. Similarly, the challenge of generating general selection rules from this information is significant. In implementations that utilised more symbolic search parameters this challenge has more obvious solutions. However, the generation of selection rules from purely numerical data without introducing high levels of complexity (and hence reducing search efficiency) requires considerable study.

The memory model proposed in this paper makes a significant step in using truly adaptive memory in a tabu search implementation. The memory model is significant step away from the current evolving memory structures and introduces the capacity for the method to learn about the solution space and explore the space intelligently on the basis of what has been learned.

\section{Conclusions}

This paper has described a potential enhancement to a tabu search algorithm for solving engineering design problems that involve continuous, real number variables. Whilst the contribution of the current paper is limited, it sets out a framework for future research to form on an understanding of human memory systems with the aim to create an extra layer of intelligence and adaptiveness in the search method. This will lead to an increase in the efficiency of the search in complex problems by allowing the method to explicitly learn about the solution space. Whilst progress to date has involved on the integration of the recency and frequency based memories, current work is focused on defining "key events" using generic concepts suitable for optimization problems using real variables. Once a key event can be captured in relation to the parameter values and outcomes, then generalised rules can be generated to limit the number of solutions in each locale to be evaluated, which will lead to a considerably more efficient search algorithm.

\section{References}

[1] Glover, F. “Tabu search (Part I)”, ORSA Journal on Computing, Vol. 1, No. 3, pp 190-206, 1989

[2] Glover, F. “Tabu search (Part II)”, ORSA Journal on Computing, Vol. 2, No. 1, pp 4-32, 1990

[3] Connor, A.M. and Tilley, D.G. “A Tabu search method for the optimisation of fluid power circuits” IMechE Journal of Systems and Control, Vol. 212, No. 5, pp 373-381, 1998

[4] Connor, A.M. and Leonard, P.J. "Pole shape optimization using a tabu search scheme”, IEEE Transactions on Magnetics, Vol. 36, No. 4, pp 1115-1118, 2000

[5] Connor, A.M., Clarkson, P.J., Shahpar, S. and Leonard, P. "Engineering design optimisation using tabu search", Proceedings of Design for Excellence: Engineering Design Conference (EDC 2000), pp371-378, 2000

[6] Matlin, M. W. “Cognition” (4th edition), Hartcourt Brace College Publishers, 1998

[7] Benjamin, L.T.H., Roy, J., \& Nation, J.R. "Psychology" (3rd edition), Macmillan College Publishing Company, 1994

[8] Tulving, E. "Episodic and semantic memory", in E. Tulving \& W. Donaldson (Eds.), Organization of memory, pp. 381-403, Academic Press, 1972

[9] Tulving, E. "Varieties of consciousness and levels of awareness in memory", in A. Baddeley \& L. Weiskrantz (Eds.), Attention: Selection, Awareness, and Control, pp. 283-300, Oxford University Press, 1993 